\title{
The Sagittal Parameters and Efficacy of Pedicle Subtraction Osteotomy in Patients with Ankylosing Spondylitis and Kyphosis Under Different Lumbar Sagittal Morphologies
}

This article was published in the following Dove Press journal:

International Journal of General Medicine

\section{Peng-Chao Zhang \\ Qiang Deng $\mathbb{D}$ \\ Wei-Bin Sheng \\ Hai-Long Guo \\ Mardan Mamat \\ Yun-Xiao Luo \\ Shu-Tao Gao}

Department of Spine Surgery, The First Affiliated Hospital of Xinjiang Medical University, Urumqi, 830054, People's Republic of China
Correspondence: Qiang Deng

Department of Spine Surgery, The First Affiliated Hospital of Xinjiang Medical

University, No. 137 of Liyushan South

Road, Xinshi District, Urumqi, 830054,

People's Republic of China

Tel +86 13325636562

Email dengqiang_abc@163.com
Objective: This study aimed to compare the changes in sagittal parameters and the efficacy of pedicle subtraction osteotomy (PSO) in patients with ankylosing spondylitis (AS) and kyphosis under different lumbar sagittal morphologies and to explore the effect of sagittal morphology on the selection of PSO levels.

Methods: A total of 24 patients with AS and thoracolumbar kyphosis (TK) who were admitted to the First Affiliated Hospital of Xinjiang Medical University between 2008 and 2019 were enrolled in this study. They were divided into two groups: a lumbar lordosis group $(\mathrm{n}=14)$ and a lumbar kyphosis group $(\mathrm{n}=10)$. Changes in sagittal parameters, lumbar Japanese Orthopaedic Association (JOA) scores, and visual analog scale (VAS) scores for lumbar pain before and after operation were compared between the two groups to evaluate postoperative efficacy.

Results: The preoperative lumbar lordosis (LL) was $-29.29 \pm 5.40$ (lordosis) and $13.50 \pm$ 3.65 (kyphosis) $(P<0.01)$, and the preoperative sagittal vertical axis (SVA) was $171.35 \pm$ 25.46 (lordosis) and $223.58 \pm 21.87$ (kyphosis) $(P<0.01)$. Preoperative global kyphosis (GK) was $75.71 \pm 5.26$ (lordosis) and $86.30 \pm 10.32$ (kyphosis) $(P<0.05)$. All patients in the lordosis group underwent PSO surgery at the twelfth thoracic vertebra (T12) or the first lumbar spinal vertebra (L1), while all patients in the kyphosis group underwent the surgery at the second or third lumbar spinal vertebra (L2 or L3). The differences in postoperative GK, LL, and SVA between the two groups were not significant $(P>0.05)$. The JOA scores of the two groups increased from $13.00 \pm 0.83$ (lordosis) and $11.30 \pm 0.93$ (kyphosis) before surgery to $21.00 \pm 0.67$ and $19.70 \pm 0.60$ after surgery $(P<0.05)$.

Conclusion: Preoperative lumbar sagittal morphology needs to be considered when selecting the optimal osteotomy plane. An osteotomy can achieve the greatest success in patients with lumbar kyphosis at L2/L3; for patients with lumbar lordosis, it can achieve satisfactory outcomes at T12/L1.

Keywords: ankylosing spondylitis, kyphosis, sagittal parameters, PSO, osteotomy

\section{Introduction}

Ankylosing spondylitis (AS) is a chronic inflammatory disease characterized by invasion of the axial spine. Approximately 30\% of sufferers will develop severe kyphosis ${ }^{1}$ and may go on to develop intra-abdominal complications. ${ }^{2-5}$ For patients with AS and kyphosis, the main purpose of surgical intervention is to reconstruct sagittal balance and restore horizontal gaze function. ${ }^{2-5}$ In recent years, pedicle 
subtraction osteotomy (PSO) has been used more frequently in clinical practice and has been found to achieve satisfactory correction and sagittal recovery results and to significantly improve the quality of life of these patients. $^{6-10}$

Choosing the most suitable osteotomy plane is a key factor in achieving good results with PSO. Jackson et al ${ }^{11}$ reported that the osteotomy plane has a $2 / 3$ angle focused on the fourth lumbar vertebra to the sacral base (L4-S1) for patients with AS and thoracolumbar kyphosis (TK). Roussouly et $\mathrm{al}^{12}$ studied four spinal features in healthy people and found that lumbar lordosis mainly relies on the third to fifth lumbar spinal vertebra (L3-L5). When considering the problem of upper and lower fixation of the osteotomy plane, it is more appropriate to undertake osteotomy at L3 or L4, as it can restore the physiological lordosis of the lumbar vertebrae. However, Qian ${ }^{13}$ and Chen et $\mathrm{al}^{6}$ have pointed out that when the apex of the kyphosis is located in the thoracolumbar segment, the osteotomy should be carried out at the apex of the kyphosis. The most successful corrective effect of osteotomy is usually achieved at the first lumbar spinal vertebra (L1). Moreover, Liu et $\mathrm{al}^{14}$ stated that osteotomy is not recommended at L3 because it reduces the patient's standing function. Another study ${ }^{15}$ of PSO surgery for the treatment of AS with TK has indicated that a lower osteotomy level can achieve more effective sagittal correction due to the leverage.

Although PSO has been extensively discussed in previous literature, there is no definitive conclusion on the optimal osteotomy level of the surgery. Previous studies ${ }^{15}$ have revealed that surgical decision making as to the osteotomy level depends largely on preoperative lumbar sagittal morphology. However, it has also been reported that pelvic incidence (PI) becomes mismatched with lumbar lordosis (LL) after PSO surgery. ${ }^{16}$ The conclusions drawn in the literature are sometimes inconsistent; therefore, this study aimed to investigate the lumbar sagittal morphology of patients with AS, with emphasis on the effect of lumbar sagittal morphology on the selection of the most effective osteotomy plane for PSO surgery.

\section{Patients and Methods}

\section{Inclusion and Exclusion Criteria}

The cases of 24 patients with AS and TK who were admitted to the First Affiliated Hospital of Xinjiang Medical University between 2008 and 2019 were retrospectively analyzed. All AS diagnoses were based on the revised New York Standard. ${ }^{17}$

Inclusion criteria: (1) Patient had been diagnosed with AS and kyphosis by symptoms, signs, and imaging examination; (2) patient had undergone PSO; (3) patient had undergone an anteroposterior and lateral film of the whole spine before and after surgery; (4) patient had at least one year of follow-up.

Exclusion criteria: (1) Kyphosis was caused by other disease factors; (2) AS was complicated by pathological fractures; (3) AS was complicated by cervical or thoracic kyphosis (in patients with lumbar lordosis, the main pathology was thoracic); (4) patient had incomplete follow-up materials.

Grouping: The lumbar sagittal morphology in the whole-spine lateral radiographs was observed. The patients with different lumbar sagittal morphology were divided into two groups based on the LL Cobb angle: a lumbar lordosis group (LL $<0^{\circ} ; \mathrm{n}=14$ ) and a lumbar kyphosis group (LL $\left.>0^{\circ} ; \mathrm{n}=10\right)$.

The study was conducted in accordance with the Declaration of Helsinki (2013 revision) and approved by the Ethics Committee of the First Affiliated Hospital of Xinjiang Medical University (NO.K202010-17). Informed consent was obtained from all patients.

\section{Procedure}

Theoretically, in patients with AS and vertebral fusion up and down, setting is not easy to open; SPO bone cutting should not be used, as forcibly cutting the bone has a high risk of vascular complications, such as nerve damage. Three-dimensional reconstruction using CT scans showed lumbar anterior column ossification and incomplete fusion, so the front is a activity, through posterior column shortening can be finished in front of open, so we are in the L5 and S1, L4 and L5, L1 and L2 SPO osteotomy, use bit except to cut bone rongeur clearance of spinous process and ligament between the spine, spine, and remove the bilateral joints. Then, the lamina, ligamentum flavum and superior articular process were bit off with lamina osteotomy forceps, and the pedicle osteotomy was retained. Finally, the posterior column was compressed to open the front and the sagittal shape of the lumbar lordosis was corrected and restored.

\section{Data Evaluation}

Surgimap software was used to measure the preoperative and postoperative sagittal vertical axis (SVA), global kyphosis (GK), PI, pelvic tilt (PT), sacral slope (SS), LL 
A

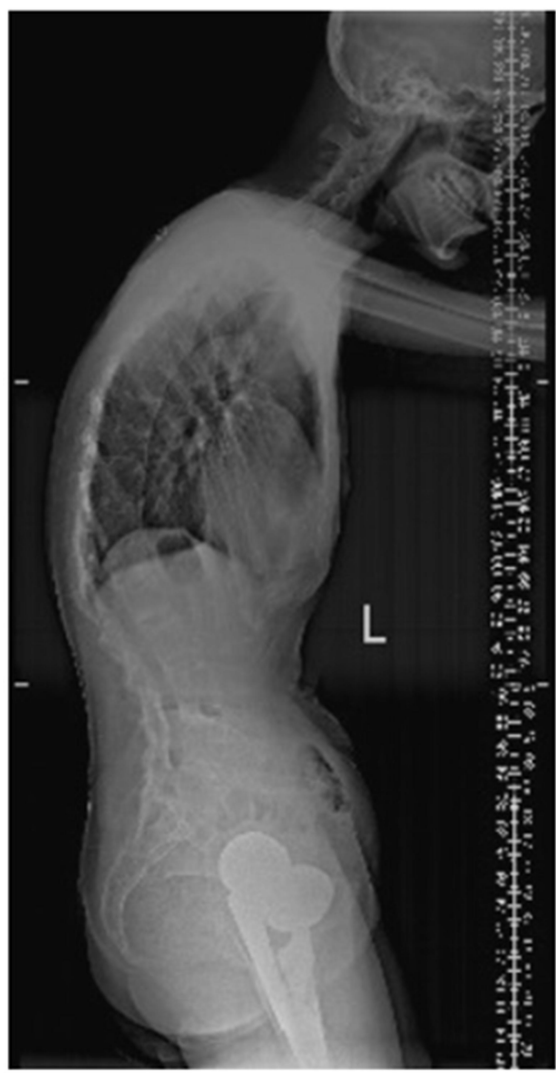

B

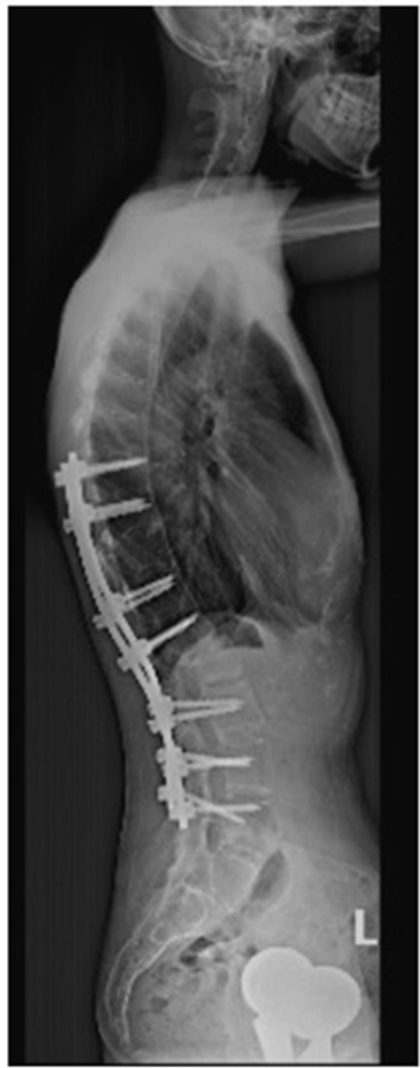

C

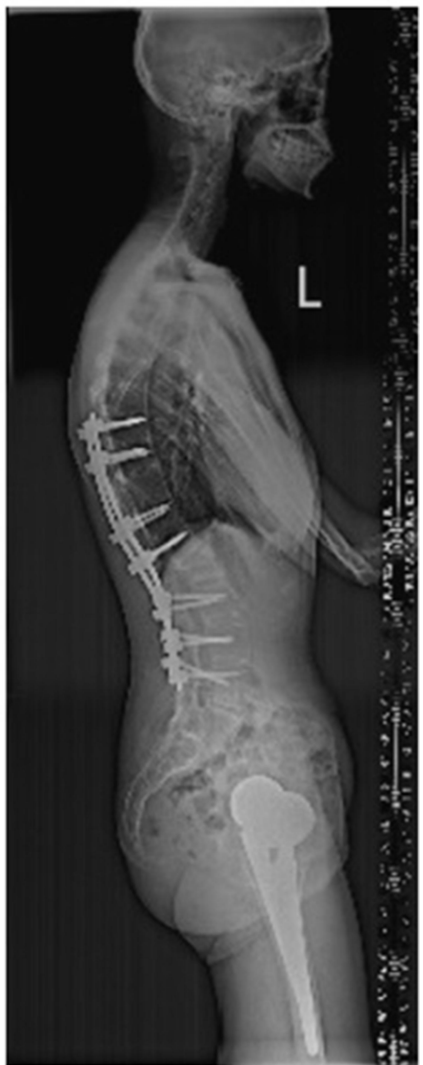

Figure I A 42-year-old female patient. (A) It shows the patient is allocated to the lumbar lordosis group before the operation. (B) Single segmental PSO surgery is performed at LI. The LL and SVA change to $-59^{\circ}$ and $38.26 \mathrm{~mm}$ from $-24^{\circ}$ and $148.73 \mathrm{~mm}$, respectively. (C) During the follow-up, no obvious correction loss is observed in the final year.

(negative value: lordosis, positive value: kyphosis), and sagittal parameters in the lateral X-ray of the whole spine when standing up. Clinical data, including age, sex, lumbar Japanese Orthopaedic Association (JOA) scores, visual analog scale (VAS) scores for lumbar pain, and complications, were reviewed at the final follow-up.

\section{Statistical Analysis}

All data were calculated using SPSS v.22 software. A paired sample $t$-test was used to compare preoperative and postoperative data and final follow-up results. An independent sample $t$-test was used to determine the difference in radiological outcomes between the two groups. $P<0.05$ was considered statistically significant.

\section{Results}

A total of 24 AS patients were enrolled from 2008 to 2019, including 14 patients in the lumbar lordosis group with a mean age of 39.2 years and ten patients in the lumbar kyphosis group with a mean age of 40.1 years. The followup time of patients in the two groups was 12 months. The PSO osteotomy was performed at T12 in seven patients and L1 in seven patients in the lordosis group. The PSO osteotomy was performed at L2 in five patients and at L3 in five patients in the kyphosis group (typical cases are shown in Figures 1-4). The postoperative sagittal parameters were significantly improved in both groups (Table 1). The mean SVA of the two groups changed from $171.35 \pm 25.46$ and $223.58 \pm 21.87$ before the operation to $55.46 \pm 5.15$ and $66.58 \pm 5.81$ after the operation, and the difference was not significant $(\mathrm{P}>0.05)$ between the two groups. The GK was corrected to $51.43 \pm 1.92$ after the operation in the lordosis group from $75.71 \pm 5.26$ before the operation, and to $52.30 \pm 3.17(\mathrm{P}<0.05)$ from $86.30 \pm 10.32$ in the kyphosis group. All sagittal parameters (GK, LL, SVA, PT, and SS) were significantly improved $(\mathrm{P}<0.05)$. No significant correction deletion was observed in the final follow-up. The difference in 
A

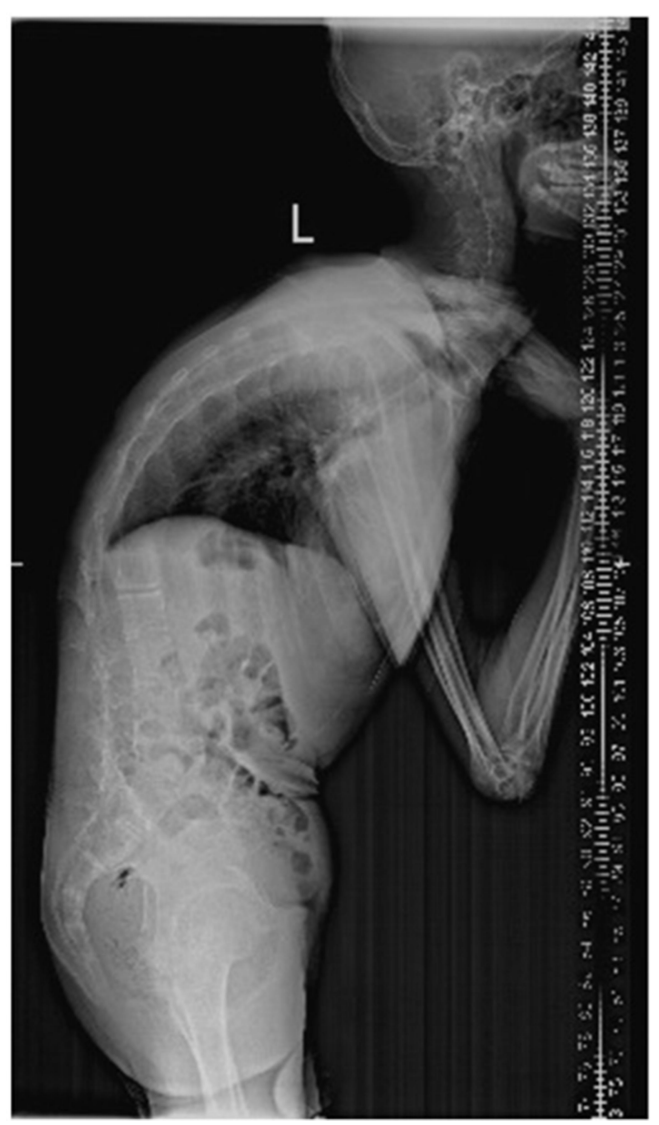

B

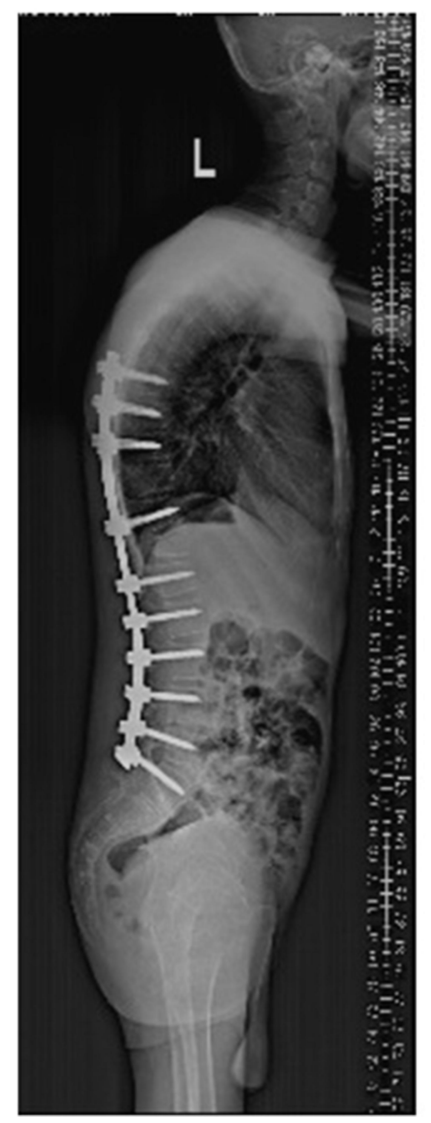

C

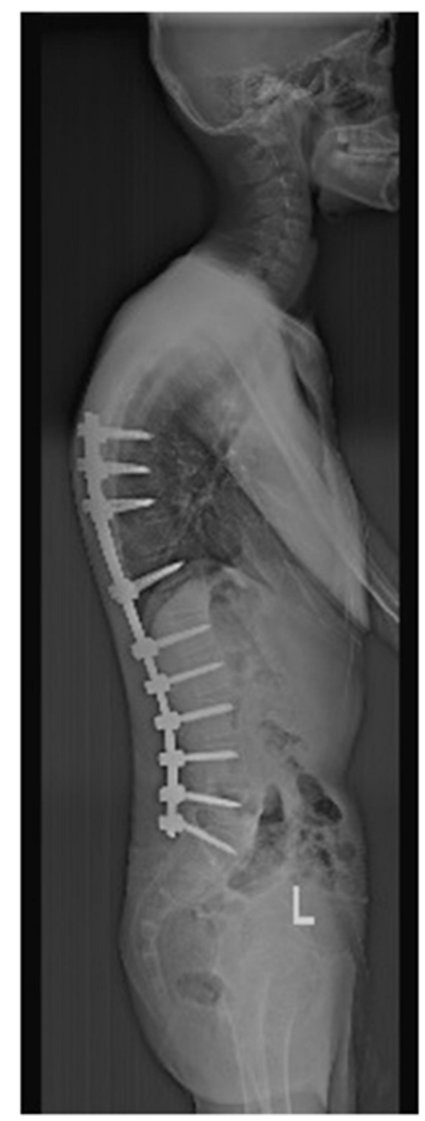

Figure 2 A 49-year-old male patient. (A) It shows the disease is located at the apex of TII-I2 before the operation, and the patient is allocated to the lumbar kyphosis group. (B) Single segmental PSO surgery is performed at TI2. The GK and SVA are corrected to $61^{\circ}$ and $72.31 \mathrm{~mm}$ from $93^{\circ}$ and $274.29 \mathrm{~mm}$, respectively. (C) During the follow-up, the GK increases to 57 , and the LL is improved to $-56^{\circ}$ from $-28^{\circ}$ in the final year. No obvious correction loss is observed.

LL, SVA, and SS was not significant $(\mathrm{P}>0.05)$ when comparing the imaging results between the two groups. The correction rate of LL and SVA in the kyphosis group was significantly higher than that in the lordosis group (P $<0.05)$. The mean postoperative JOA scores of the two groups increased from $11.30 \pm 0.93$ to $23.00 \pm 0.68$, and the VAS scores improved from $7.05 \pm 0.39$ to $1.87 \pm 0.13$, and the difference was not significant $(\mathrm{P}>0.05)$ between the lordosis and kyphosis groups.

Table 2 shows the preoperative sagittal parameters of four groups (T12, L1, L2, and L3). The GK, LL, and SS of the preoperative L2 and L3 groups are significantly higher than those of the T12 and L1 groups $(\mathrm{P}<0.05)$.

Table 3 summarizes the clinical effects. The mean value of postoperative JOA increases to 22.39 from 11.15, and the VAS scores decrease to 1.72 from 7.99. There is no significant difference between subgroups $(\mathrm{P}<0.05)$.

\section{Discussion}

The key factor in surgical decision making for AS complicated with TK is restoring the sagittal balance of the patient. Previous studies ${ }^{15,18}$ have indicated that LL is correlated with PT, PI, and SS to some extent, while TK is not significantly correlated with them. Legaye et al ${ }^{19}$ proposed that LL and SS are most closely related to scoliosis $(r=0.80)$ and that pelvic parameters can be used to predict LL values. Gottfried et $\mathrm{al}^{20}$ found that the PI and PT values of patients with flatback deformity after spinal osteotomy were significantly higher than those of patients without flatback deformity. They speculated that the cause of flatback deformity may be related to a higher preoperative PI value in this group of patients. They also suggested that patients with high preoperative PI values may not be able to tolerate LL loss, which further suggests that PI and LL should be used as important reference 
A

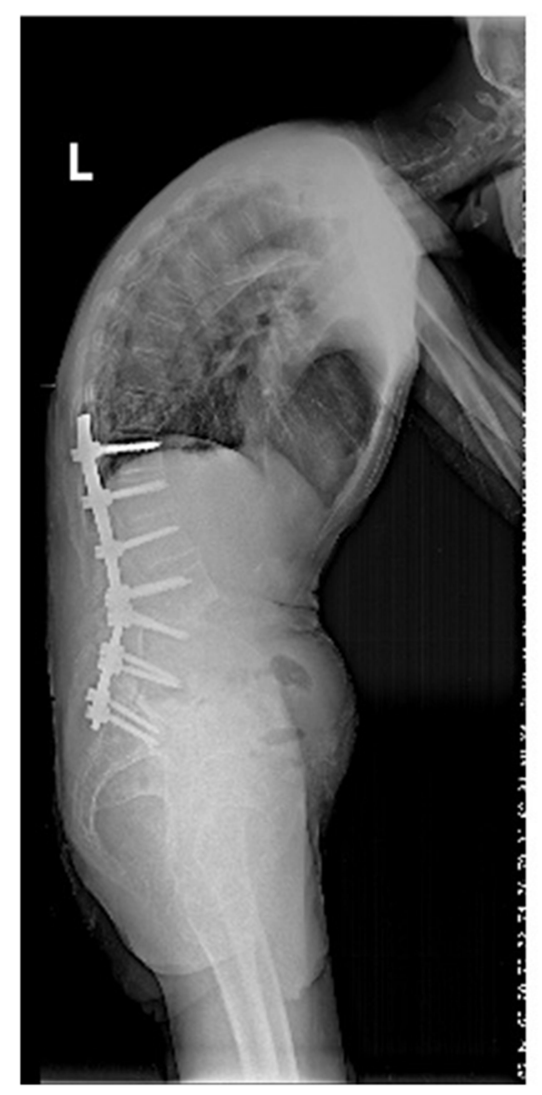

B

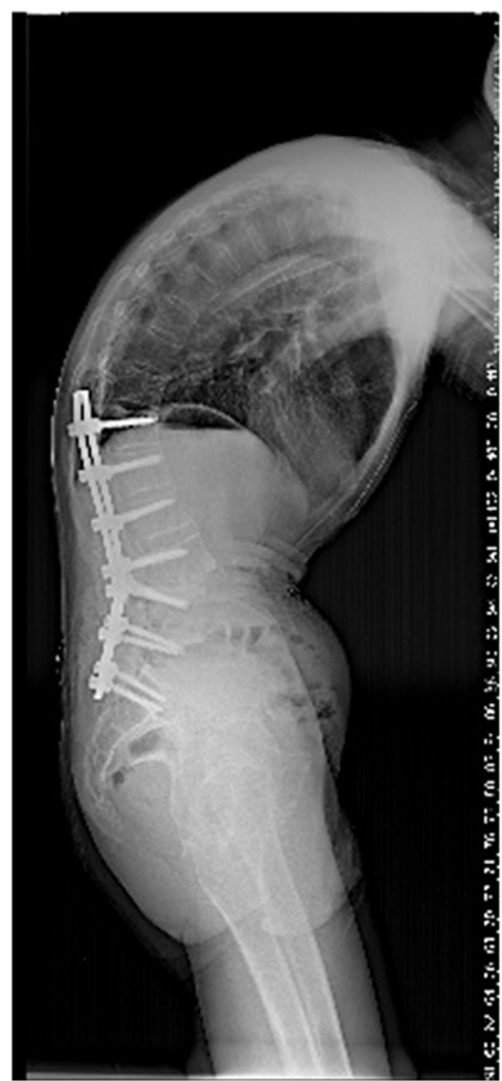

C

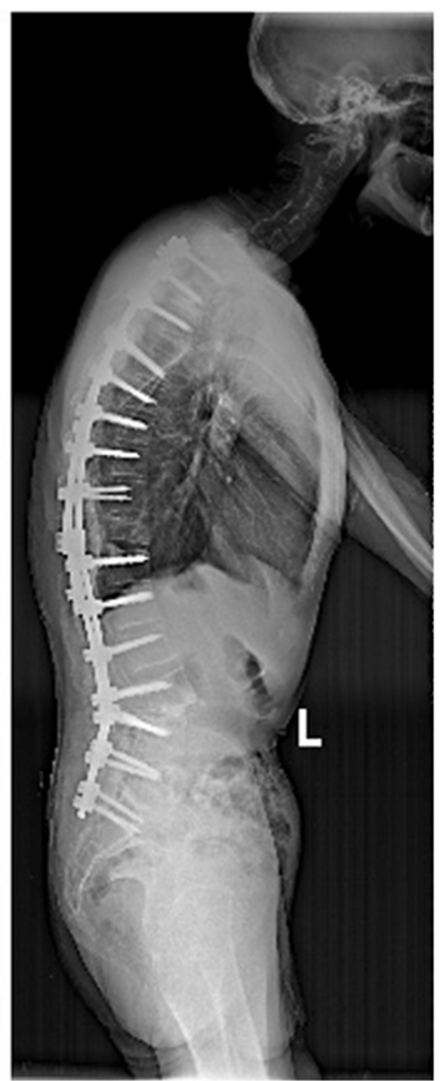

Figure 3 A 49-year-old male patient. (A) The patient is allocated to the lumbar kyphosis group before the operation. (B) Single segmental PSO surgery is performed at L2. The $L L$ and SVA change to $-56^{\circ}$ and $63.06 \mathrm{~mm}$ from $8^{\circ}$ and $312.84 \mathrm{~mm}$, respectively (C). During the follow-up, no significant correction loss is observed in the final year. The $\mathrm{LL}$ and SVA are $-56^{\circ}$ and $70.35 \mathrm{~mm}$, respectively.

indicators for the preoperative evaluation of adult patients with spinal deformities. Hence, it is necessary to study the sagittal morphology of the lumbar vertebrae, using LL as a guide.

Thomasen first proposed PSO as a method of correcting AS with severe kyphosis in 1985. The method can achieve a $30^{\circ}-35^{\circ}$ correction of deformity ${ }^{21,22}$ through a V-osteotomy of the pedicle, using the anterior cortical bone of the vertebral body as the correction axis and closing the posterior column to close the wedge gap at the osteotomy of the anterior middle column. Because the anterior column is not open, the tension of the anterior vertebrae tissue in the orthosis is small, thereby reducing the risk of anterior longitudinal ligament and abdominal aortic tear. Many studies have reported ${ }^{23,24}$ that PSO can effectively restore LL and SVA to reconstruct sagittal balance. However, the procedure is also associated with complications, ${ }^{23}$ such as nerve and vascular injuries. Despite this, it has become the most commonly used surgical method for correcting TK deformity in patients with AS. The present study found that the average correction degree of PSO in 24 patients with AS and TK was $30.3^{\circ}$, which is consistent with previous studies.

This study investigated the different sagittal patterns of the lumbar vertebrae in patients with AS and TK. Satisfactory correction was achieved in all 24 patients after surgery. The 14 patients in the lumbar lordosis group underwent PSO at the twelfth thoracic vertebra (T12) or the first lumbar spinal vertebra (L1), while the 10 in the lumbar kyphosis group underwent PSO at the second or third lumbar spinal vertebra (L2 or L3). A slight loss $\left(1.4^{\circ}-2.7^{\circ}\right)$ of correction degree was identified in both groups at the last follow-up. This is similar to the results of $\mathrm{Liu}^{14}$ and Qian. ${ }^{25}$

For the calculation of osteotomy angles, Surgimap software was used for preoperative simulation (Figure 5) in all patients in the present study. Osteotomy angle = preoperative maxillary eyebrow angle + preoperative PT-tPT-10 
A

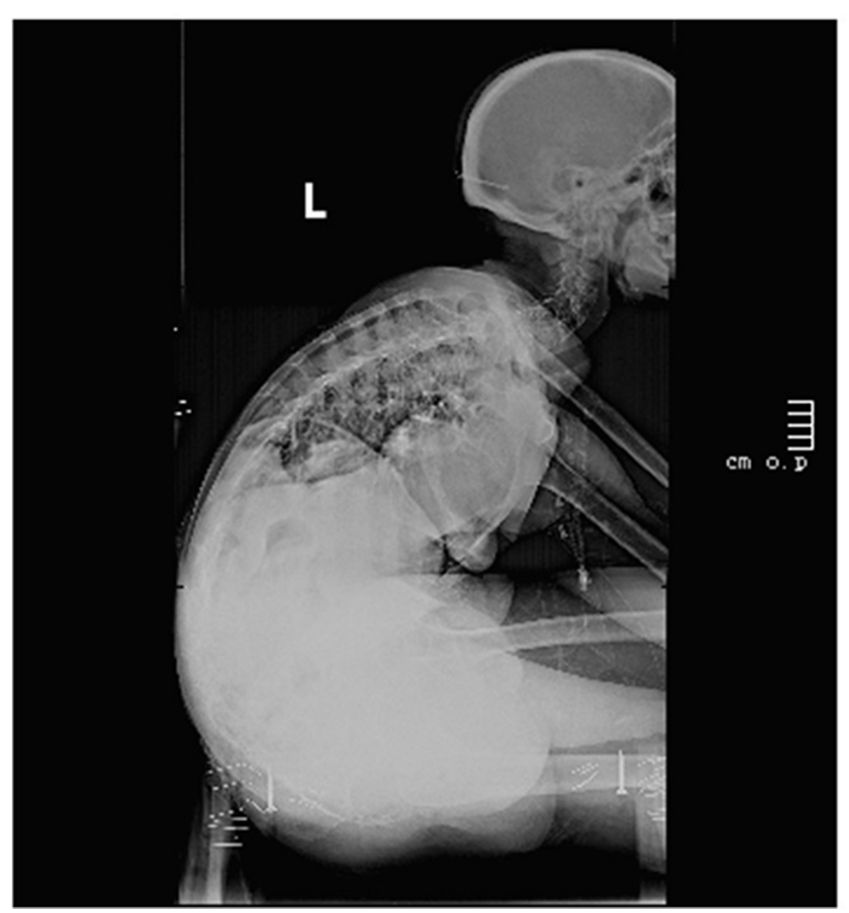

B

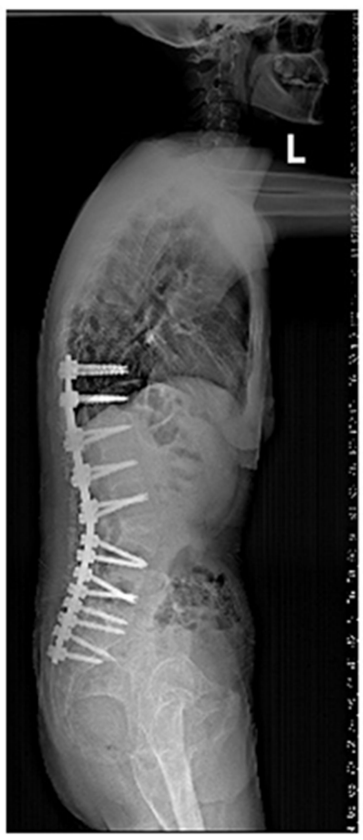

C

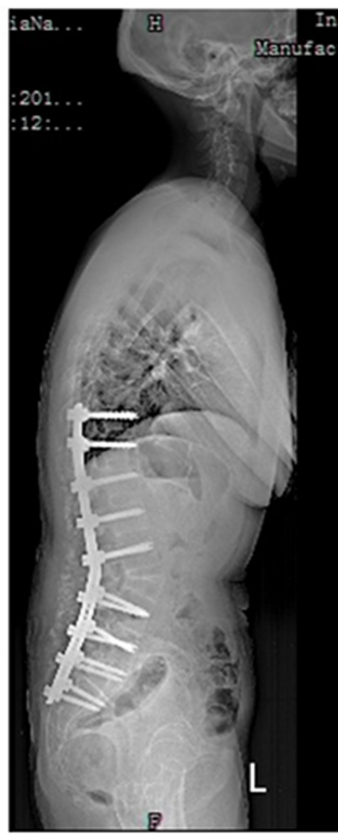

Figure 4 A 46-year-old female patient. (A) It shows the disease is located at the apex of L2-L3, and the patient is allocated to the lumbar kyphosis group. (B) The SPO osteotomy is performed at LI-L2, L4-L5, and L5-SI. The single segmental PSO osteotomy is performed at L3. The GK and SVA change to $59^{\circ}$ and 56.36 mm from $103^{\circ}$ and $261.49 \mathrm{~mm}$, respectively. The LL is corrected to postoperative $-44^{\circ}$ from preoperative $41^{\circ}$. (C) During the follow-up, no significant correction loss is observed in the final year. The LL, GK, and SVA are $-45^{\circ}, 62^{\circ}$, and $48.82 \mathrm{~mm}$, respectively.

(tPT is an ideal PT value; $\mathrm{tPT}=0.37 \times \mathrm{PT}-7$ ). The preoperative theoretical osteotomy degree was between $31.5^{\circ}$ and $33^{\circ}$ in the lumbar lordosis group and between $32.5^{\circ}$ and $82.5^{\circ}$ in the kyphosis group. Previous studies have reported an average corrective degree of $16^{\circ},{ }^{26} 35^{\circ},{ }^{24}$ and $38^{\circ 27}$ for single-segmental PSO in patients with AS and an improvement of $30-130 \mathrm{~mm}^{28}$ after surgery. The results of the present study are consistent with these findings: the average $\mathrm{GK}$ and $\mathrm{LL}$ correction values were $37.53^{\circ}$ and $29.51^{\circ}$, respectively, and the SVA correction was about $116.96 \mathrm{~mm}$. There was also no obvious loss within a one-year follow-up period, which is largely consistent with the calculation of preoperative theoretical values. The preoperative SVA and GK of patients in the kyphosis group were obviously worse when comparing the preoperative radiological parameters of the two groups, and the postoperative JOA and VAS scores of patients in the kyphosis group were lower than those in the lordosis group.

In addition, five patients with lumbar kyphosis underwent total hip arthroplasty (THA) before PSO. There is still controversy about the order of surgery for patients with AS and TK: should THA be undertaken first to address AS hip joint deformity, or should spinal orthopedic surgery be undertaken first to treat kyphosis deformity? Le et $\mathrm{al}^{29}$ believed that performing a spinal osteotomy first, following by THA, allows more accurate determination of the acetabular anterior inclination and abduction angles. Guan et $\mathrm{al}^{30}$ stated that undertaking spinal posture surgery after THA surgery is more convenient because the spinal orthopedic surgery can be performed with the patient prone. Vaz and Roussouly et $\mathrm{al}^{31}$ found that PT and TK values decreased after THA when AS affected the hip joints, while SS and LL values increased. As previous findings suggest that spinal and pelvic parameters are improved by undertaking THA first, five of the patients in the present study underwent THA before spinal surgery.

In this study, all patients in the kyphosis group underwent PSO surgery at L2 or L3. The osteotomy at L3 could provide a larger range of bone resection and longer PSO leverage than the upper lumbar vertebrae and achieve maximum correction of LL and SVA. However, such patients often require sacral base (S1) fixation. For precise control in PSO surgery, orthodontic, in drawing up bone cutting cone and two sections near the cone into long 
Table I Comparison of Lordosis (A) and Kyphosis (B) Groups

\begin{tabular}{|c|c|c|c|}
\hline Variate & A Group & B Group & $P$ \\
\hline \multicolumn{4}{|l|}{ LL } \\
\hline Before the operation & $-29.29 \pm 5.40$ & $13.50 \pm 3.65$ & $<0.001$ \\
\hline After the operation & $-44.86 \pm 3.20 *$ & $-41.00 \pm 3.01 *$ & 0.408 \\
\hline One year after the operation & $-44.29 \pm 1.88^{*}$ & $-43.10 \pm 2.80 *$ & 0.718 \\
\hline \multicolumn{4}{|l|}{ SVA } \\
\hline Before the operation & $|7| .35 \pm 25.46$ & $223.58 \pm 21.87$ & 0.155 \\
\hline After the operation & $54.39 \pm 6.35^{*}$ & $64.59 \pm 6.09 *$ & 0.276 \\
\hline One year after the operation & $55.46 \pm 5.15^{*}$ & $66.58 \pm 5.8 I^{*}$ & 0.170 \\
\hline \multicolumn{4}{|l|}{ GK } \\
\hline Before the operation & $75.7 I \pm 5.26$ & $86.30 \pm 10.32$ & 0.332 \\
\hline After the operation & $52.00 \pm 1.75^{*}$ & $51.30 \pm 2.75^{*}$ & 0.823 \\
\hline One year after the operation & $51.43 \pm 1.92 *$ & $52.30 \pm 3.17^{*}$ & 0.806 \\
\hline \multicolumn{4}{|l|}{ PT } \\
\hline Before the operation & $38.29 \pm 3.79$ & $36.80 \pm 5.02$ & 0.812 \\
\hline After the operation & $28.43 \pm 3.08^{*}$ & $25.40 \pm 4.46 *$ & 0.569 \\
\hline One year after the operation & $25.07 \pm 2.74 *$ & $23.00 \pm 3.65^{*}$ & 0.648 \\
\hline \multicolumn{4}{|l|}{ PI } \\
\hline Before the operation & $64.29 \pm 4.58$ & $50.80 \pm 5.38$ & 0.070 \\
\hline After the operation & $62.21 \pm 4.46$ & $53.40 \pm 3.88$ & 0.171 \\
\hline One year after the operation & $61.64 \pm 4.07$ & $54.40 \pm 4.00$ & 0.232 \\
\hline \multicolumn{4}{|l|}{ SS } \\
\hline Before the operation & $25.86 \pm 2.13$ & $14.00 \pm 2.87$ & 0.003 \\
\hline After the operation & $33.50 \pm 2.34 *$ & $28.00 \pm 1.67^{*}$ & 0.091 \\
\hline One year after the operation & $36.29 \pm 1.77^{*}$ & $31.40 \pm 1.96 *$ & 0.081 \\
\hline \multicolumn{4}{|l|}{ VAS } \\
\hline Before the operation & $7.80 \pm 0.25$ & $7.05 \pm 0.39$ & 0.104 \\
\hline After the operation & $3.13 \pm 0.23 *$ & $3.02 \pm 0.25^{*}$ & 0.751 \\
\hline One year after the operation & $1.87 \pm 0.13^{*}$ & $1.58 \pm 0.14^{*}$ & 0.132 \\
\hline \multicolumn{4}{|l|}{ JOA } \\
\hline Before the operation & $13.00 \pm 0.83$ & $11.30 \pm 0.93$ & 0.189 \\
\hline After the operation & $21.00 \pm 0.67^{*}$ & $19.70 \pm 0.60 *$ & 0.182 \\
\hline One year after the operation & $22.86 \pm 0.60 *$ & $23.00 \pm 0.68^{*}$ & 0.878 \\
\hline
\end{tabular}

Note: Compared with before the operation, ${ }^{*} \mathrm{P}<0.001$.

Table 2 Preoperative Comparison of Four Subgroups (TI2, LI, L2 and L3)

\begin{tabular}{|l|l|l|l|l|l|}
\hline Variate & Group TI2 & Group LI & Group L2 & Group L3 \\
\hline LL & $-37.29 \pm 23.16$ & $-21.29 \pm 5.34$ & $11.00 \pm 4.03^{*}$ & $16.00 \pm 6.37^{*}$ \\
SVA & $184.91 \pm 118.73$ & $157.79 \pm 27.10$ & $194.74 \pm 33.78$ & $252.42 \pm 24.39 *$ & $<0.001$ \\
GK & $85.86 \pm 18.38$ & $65.57 \pm 6.11$ & $63.40 \pm 10.44$ & $109.20 \pm 10.38^{*}$ & 0.339 \\
PT & $35.29 \pm 15.52$ & $41.29 \pm 4.98$ & $33.60 \pm 6.80$ & $40.00 \pm 7.87$ & 0.004 \\
PI & $65.43 \pm 18.5$ & $63.41 \pm 6.44$ & $47.60 \pm 9.41$ & $54.00 \pm 6.05$ & 0.792 \\
SS & $29.86 \pm 5.08$ & $21.86 \pm 3.28$ & $14.00 \pm 4.10$ & $14.00 \pm 4.49$ & 0.318 \\
VAS & $7.62 \pm 1$ & $7.99 \pm 0.35$ & $6.75 \pm 0.51$ & $7.34 \pm 0.62$ & 0.008 \\
JOA & $14.86 \pm 1.77$ & $11.14 \pm 1.16$ & $11.20 \pm 0.66$ & $11.40 \pm 1.86$ & 0.305 \\
\hline
\end{tabular}

Note: Compared with other group, ${ }^{*} \mathrm{P}<0.01$. 
Table 3 Efficacy Comparison of Four Subgroups (TI2, LI, L2 and L3)

\begin{tabular}{|l|l|l|l|l|l|}
\hline Variate & Group TI2 & Group LI & Group L2 & Group L3 & P \\
\hline VAS & & & & \\
$\quad$ Before the operation & $7.62 \pm 1$ & $7.99 \pm 0.94$ & $6.75 \pm 1.13$ & $7.34 \pm 1.39$ & 0.305 \\
After the operation & $3.07 \pm 0.84$ & $3.19 \pm 0.96^{*}$ & $2.81 \pm 1.07^{*}$ & $3.23 \pm 0.32^{*}$ & 0.850 \\
One year after the operation & $1.76 \pm 0.52$ & $1.99 \pm 0.44^{*}$ & $1.50 \pm 0.45^{*}$ & $1.66 \pm 0.44^{*}$ & 0.354 \\
\hline JOA & & & & $11.40 \pm 4.16$ \\
Before the operation & $14.86 \pm 1.77$ & $11.14 \pm 3.08$ & $11.20 \pm 1.48$ & $19.00 \pm 1.58^{*}$ \\
After the operation & $22.57 \pm 2.23$ & $19.43 \pm 1.72^{*}$ & $20.40 \pm 2.07^{*}$ & 0.065 \\
One year after the operation & $24.43 \pm 1.81$ & $21.29 \pm 1.38^{*}$ & $23.20 \pm 2.68^{*}$ & $22.80 \pm 1.79^{*}$ \\
\hline
\end{tabular}

Notes: There was no significant difference in scores among the three groups; JOA score of L3 was compared with the first three groups $(P<0.05)$; Compared with after the operation, $* \mathrm{P}<0.0 \mathrm{l}$.

segmental pedicle screws and install a temporary fixed rod (that is, the four fixed adjacent cones in order to maintain the stability of the spine). Longer segmental internal fixation can effectively maintain the protrusion deformity after correction, so AS patients often experience vertebral osteoporosis. Strong fixation of the long segmental pedicle screw can avoid loosening and emergence of the postoperative screw and prevent the need for postoperative

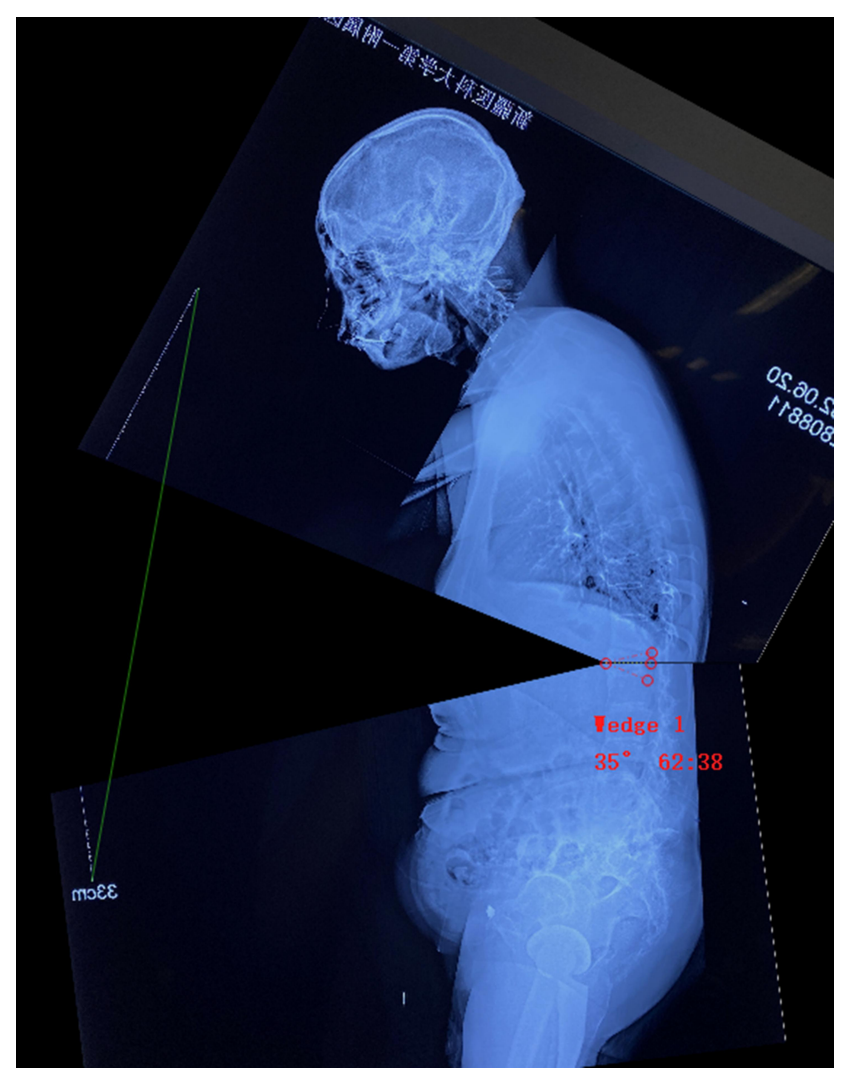

Figure 5 Simulated selection of osteotomy plane and PSO angle before operation. Simulated osteotomy angle $=$ preoperative mandibular eyebrow angle + preoperative PT-tPT-10, tPT $=0.37 \times$ PT-7. convex deformity correction after failure. To formulate the cone, a V-shaped cut is made on the bone according to the required angle of bone cutting. Titanium rods are bent to the shape of the reset bone and the bed is slowly adjusted to reset the spine. Gradually closed bone cutting clearance, maintaining the dural sac and nerve-root compression, evoked potential amplitude detection continuously. In order to address abnormal situations in a timely manner, a closed complete intraoperative X-ray should be taken to confirmed reset and no significant deviation.

For patients with severe kyphosis, when the required correction exceeds $60^{\circ}$, two-stage PSO or PSO + SmithPeterson osteotomy (SPO) should be considered to obtain a larger range of correction. In the present study, a 46-year -old woman (Figure 4) presented a typical folding-knife back. Her chin-brow vertical angle (CBVA) was $-58^{\circ}$, and her SVA was approximately $300 \mathrm{~mm}$. Her lumbar spine was kyphotic, and her LL was $45^{\circ}$. An osteotomy angle of $86.5^{\circ}$ was required before the operation. $\mathrm{PSO}+\mathrm{SPO}$ was performed for this patient, using Surgimap software to simulate every individual segment that could provide a $15^{\circ}$ correction from osteotomy. Single-segmental PSO can provide a $40^{\circ}$ correction at most, while the combined surgery can provide a total of $85^{\circ}$ correction. Therefore, the SPO osteotomy was undertaken at L5-S1 and L4-L5.

In three-dimensional reconstruction from $\mathrm{CT}$ scans, the patient showed lumbar anterior column ossification and incomplete fusion, so the front is an activity, through the posterior column of shortening are able to do in front of open, so we are in the L5 and S1, L4 and L5, L1 and L2 SPO osteotomy, use bit except to cut bone rongeur clearance of spinous process and ligament between the spine, spine, and remove the bilateral joints, reoccupy lamina rongeur bite in addition to the part of the lamina, 
yellow ligament and articular process, retain pedicle bone cutting, finally open the front, compressed column to achieve correct the restoration of lumbar lordosis sagittal morphology and L1-L2 and PSO osteotomy at L3, respectively. Fixed segments were at T9-S1. The postoperative CBVA was improved to $8^{\circ}$, SVA to $48.4 \mathrm{~mm}$, and $\mathrm{LL}$ to $-44^{\circ}$. One year after the operation, no correction loss was observed in this patient. From the patient's point of view, satisfactory sagittal shape and good quality of life were achieved.

Previous studies ${ }^{6,22}$ have reported that PSO should be performed at the apex of kyphosis. However, the present study suggests that the patient's preoperative sagittal morphology needs to be evaluated if the osteotomy plane is close to the kyphotic apex. Based on the results of this study, it appears that patients with lumbar kyphosis usually present with kyphosis in the thoracolumbar segments. T12 or L1 are therefore recommended as the osteotomy plane because they are relatively close to the kyphotic apex and can therefore achieve a better correction of TK. Owing to the reduction of fixed segments, the risk of postoperative PJK can be reduced.

For patients with lumbar kyphosis, PSO is recommended at L2 or L3. The preoperative sagittal imbalance is often severe in such patients, which means a greater correction is needed to achieve a satisfactory outcome. The adult spinal cones terminate at L2, and a lower level of osteotomy can reduce the risk of spinal cord injury. However, S1 fixation is often required at L3 in patients with osteotomy, which may result in a poor quality of life. ${ }^{32}$ However, taking into consideration a better correction of LL and SVA, this is an acceptable option. Therefore, the osteotomy position of patients with lumbar kyphosis should be either L2 or L3.

\section{Conclusion}

The present study has some limitations. Firstly, it was a retrospective study. Secondly, its sample size was small, especially for patients with lumbar kyphosis. Moreover, none of the enrolled patients underwent PSO at L4. As such, larger cohort studies are needed to improve the classification of lumbar sagittal morphology in patients with AS. Lastly, the conclusion of this study is relatively preliminary because both groups lacked a control group for comparison. Further prospective cohort studies are needed to determine the optimal osteotomy level for single-level PSO.

\section{Acknowledgments}

We are particularly grateful to all the people who have given us help in this article.

\section{Funding}

This study was funded by the Research on TGF- $\beta 2$ in regulating the apoptotic signaling pathways of Fluoridestained Cells Project (No.2018D01C158). The funding body had no role in the design of the study and collection, analysis, and interpretation of data and in writing the manuscript.

\section{Disclosure}

The authors declare that they have no competing interest.

\section{References}

1. Koller H, Koller J, Mayer M, Hempfing A, Hitzl W. Osteotomies in ankylosing spondylitis: where, how many, and how much? Eur Spine J. 2018;27(Suppl 1):70-100. doi:10.1007/s00586-017-5421-z

2. Campochiaro C, Caruso PF. Ankylosing spondylitis and axial spondyloarthritis. N Engl J Med. 2016;375(13):1302. doi:10.1056/ NEJMc1609622

3. Uckun A, Sezer I. Ankylosing spondylitis and balance. Eurasian $J$ Med. 2017;49(3):207-210. doi:10.5152/eurasianjmed.2017.17116

4. QQian BP, Huang JC, Qiu Y, et al. Complications of spinal osteotomy for thoracolumbar kyphosis secondary to ankylosing spondylitis in 342 patients: incidence and risk factors. J Neurosurg Spine. 2018;30(1):91-98. doi:10.3171/2018.6.SPINE171277

5. Tominaga R, Fukuma S, Yamazaki S, et al. Relationship between kyphotic posture and falls in community-dwelling men and women: the locomotive syndrome and health outcome in Aizu cohort study. Spine (Phila Pa 1976). 2016;41(15):1232-1238. doi:10.1097/ BRS.0000000000001602

6. Chen IH, Chien JT, Yu TC. Transpedicular wedge osteotomy for correction of thoracolumbar kyphosis in ankylosing spondylitis: experience with 78 patients. Spine (Phila Pa 1976). 2001;26(16): E354-E360. doi:10.1097/00007632-200108150-00010

7. Xu H, Zhang Y, Zhao Y, Zhang X, Xiao S, Wang Y. Radiologic and clinical outcomes comparison between single- and two-level pedicle subtraction osteotomies in correcting ankylosing spondylitis kyphosis. Spine J. 2015;15(2):290-297. doi:10.1016/j. spinee.2014.09.014

8. Kim KT, Park DH, Lee SH, Lee JH. Results of corrective osteotomy and treatment strategy for ankylosing spondylitis with kyphotic deformity. Clin Orthop Surg. 2015;7(3):330-336. doi:10.4055/ cios.2015.7.3.330

9. Yıldız F, Akgül T, Ekinci M, Dikici F, Şar C, Domaniç Ü. Results of closing wedge osteotomy in the treatment of sagittal imbalance due to ankylosing spondylitis. Acta Orthop Traumatol Turc. 2016;50 (1):63-68. doi:10.3944/AOTT.2016.14.0059

10. Hua WB, Zhang YK, Gao Y, et al. Analysis of sagittal parameters in patients undergoing one- or two-level closing wedge osteotomy for correcting thoracolumbar kyphosis secondary to ankylosing spondylitis. Spine (Phila Pa 1976). 2017;42(14):E848-E854. doi:10.1097/BRS.0000000000001984

11. R P Jackson, Mcmanus AC. Pelvic lordosis and pelvic incidence: the relationship of pelvic parameters to sagittal spinal profile. Curr Opin Orthop. 2004;15(3):150-153. doi:10.1097/01.bco.0000120 $643.46244 .8 \mathrm{a}$ 
12. Roussouly P, Gollogly S, Berthonnaud E, Dimnet J. Classification of the normal variation in the sagittal alignment of the human lumbar spine and pelvis in the standing position. Spine (Phila Pa 1976). 2005;30(3):346-353. doi:10.1097/01.brs.0000152379.54463.65

13. Qian BP, Wang XH, Qiu Y, et al. The influence of closing-opening wedge osteotomy on sagittal balance in thoracolumbar kyphosis secondary to ankylosing spondylitis: a comparison with closing wedge osteotomy. Spine (Phila Pa 1976). 2012;37(16):1415-1423. doi:10.1097/BRS.0b013e318250dc95

14. Liu C, Zheng G, Guo Y, et al. Two-level osteotomy for correcting severe ankylosing spondylitis kyphosis: radiologic outcomes of different osteotomy position-selection strategy for different type of patients. Spine Deform. 2018;6(3):273-281. doi:10.1016/j.jspd.2017.10.011

15. Kim KT, Suk KS, Cho YJ, Hong GP, Park BJ. Clinical outcome results of pedicle subtraction osteotomy in ankylosing spondylitis with kyphotic deformity. Spine (Phila Pa 1976). 2002;27 (6):612-618. doi:10.1097/00007632-200203150-00010

16. Liu ZJ, Qian BP, Qiu Y, Mao SH, Jiang J, Wang B. Does postoperative PI-LL mismatching affect surgical outcomes in thoracolumbar kyphosis associated with ankylosing spondylitis patients? Clin Neurol Neurosurg. 2018;169:71-76. doi:10.1016/j.clineuro.2018.04.006

17. Mathies H. Diagnostische Kriterien der Spondylitis ankylosans [Diagnostic criteria in ankylosing spondylitis]. Dtsch Med Wochenschr. 1984;109(10):382-385. doi:10.1055/s-2008-1069200

18. Chang KW, Chen YY, Lin CC, Hsu HL, Pai KC. Closing wedge osteotomy versus opening wedge osteotomy in ankylosing spondylitis with thoracolumbar kyphotic deformity. Spine (Phila Pa 1976). 2005;30(14):1584-1593. doi:10.1097/01.brs.0000170300.17082.49

19. Legaye J, Duval-Beaupère G, Hecquet J, Marty C. Pelvic incidence: a fundamental pelvic parameter for three-dimensional regulation of spinal sagittal curves. Eur Spine J. 1998;7(2):99-103. doi:10.1007/ s005860050038

20. Gottfried ON, Daubs MD, Patel AA, Dailey AT, Brodke DS. Spinopelvic parameters in postfusion flatback deformity patients. Spine J. 2009;9(8):639-647. doi:10.1016/j.spinee.2009.04.008

21. Kim YJ, Bridwell KH, Lenke LG, Cheh G, Baldus C. Results of lumbar pedicle subtraction osteotomies for fixed sagittal imbalance: a minimum 5-year follow-up study. Spine (Phila Pa 1976). 2007;32 (20):2189-2197. doi:10.1097/BRS.0b013e31814b8371

22. Zhu Z, Wang X, Qian B, et al. Loss of correction in the treatment of thoracolumbar kyphosis secondary to ankylosing spondylitis: a comparison between Smith-Petersen osteotomies and pedicle subtraction osteotomy. J Spinal Disord Tech. 2012;25(7):383-390. doi:10.1097/BSD.0b013e318224b199
23. Enercan M, Ozturk C, Kahraman S, Sarıer M, Hamzaoglu A, Alanay A. Osteotomies/spinal column resections in adult deformity. Eur Spine J. 2013;22(Suppl 2):S254-S264. doi:10.1007/s00586-012-2313-0

24. Kim KT, Lee SH, Suk KS, Lee JH, Jeong BO. Outcome of pedicle subtraction osteotomies for fixed sagittal imbalance of multiple etiologies: a retrospective review of 140 patients. Spine (Phila Pa 1976). 2012;37(19):1667-1675. doi:10.1097/BRS.0b013e3182552fd0

25. Diao WY, Qian BP, Qiu Y, Zhu ZZ, Wang B, Yu Y. Does the preoperative lumbar sagittal profile affect the selection of osteotomy level in pedicle subtraction osteotomy for thoracolumbar kyphosis secondary to ankylosing spondylitis? Clin Neurol Neurosurg. 2018;172:39-45. doi:10.1016/j.clineuro.2018.06.026

26. Park YS, Kim HS, Baek SW, Oh JH. Preoperative computer-based simulations for the correction of kyphotic deformities in ankylosing spondylitis patients. Spine J. 2014;14(10):2420-2424. doi:10.1016/j. spinee.2014.02.022

27. Arun R, Dabke HV, Mehdian H. Comparison of three types of lumbar osteotomy for ankylosing spondylitis: a case series and evolution of a safe technique for instrumented reduction. Eur Spine J. 2011;20 (12):2252-2260. doi:10.1007/s00586-011-1894-3

28. Xin Z, Zheng G, Huang P, Zhang X, Wang Y. Clinical results and surgery tactics of spinal osteotomy for ankylosing spondylitis kyphosis: experience of 428 patients. J Orthop Surg Res. 2019;14(1):330. doi:10.1186/s13018-019-1371-y

29. Le Huec JC, Leijssen P, Duarte M, Aunoble S. Thoracolumbar imbalance analysis for osteotomy planification using a new method: FBI technique. Eur Spine J. 2011;20(Suppl 5):669-680. doi:10.1007/ s00586-011-1935-y

30. Guan M, Wang J, Zhao L, Xiao J, Li Z, Shi Z. Management of hip involvement in ankylosing spondylitis. Clin Rheumatol. 2013;32 (8):1115-1120. doi:10.1007/s10067-013-2278-3

31. Roussouly P, Transfeldt E, Schwender J, et al. 7:58 Sagittal morphology and equilibrium of pelvis and spine in normals. Spine J. 2002;2 (5):61-62. doi:10.1016/S1529-9430(02)00299-1

32. Abe H, Matsunaga A, Watanabe H, et al. Relationship between the severity of osteoarthritis of the knee and spino-pelvic alignment in Japanese patients with knee osteoarthritis. Physiotherapy. 2015;101: e29-e30. doi:10.1016/j.physio.2015.03.139
International Journal of General Medicine

\section{Publish your work in this journal}

The International Journal of General Medicine is an international, peer-reviewed open-access journal that focuses on general and internal medicine, pathogenesis, epidemiology, diagnosis, monitoring and treatment protocols. The journal is characterized by the rapid reporting of reviews, original research and clinical studies across all disease areas. The manuscript management system is completely online and includes a very quick and fair peer-review system, which is all easy to use. Visit http://www.dovepress.com/ testimonials.php to read real quotes from published authors. 